Pacific

Journal of

Mathematics

\title{
ZETA INVARIANTS FOR DIRICHLET SERIES
}

MAURo SPREAFICO

Volume $224 \quad$ No. 1

March 2006 


\title{
ZETA INVARIANTS FOR DIRICHLET SERIES
}

\author{
MAURO SPREAFICO
}

\begin{abstract}
We introduce a general method for obtaining the main zeta invariants for a class of double series of Dirichlet type and we apply it to the case of homogeneous quadratic and linear double series.
\end{abstract}

\section{Introduction and statement of the problem}

Let $n \in \mathbb{N}_{0}^{2}$, where $\mathbb{N}_{0}=\{1,2,3, \ldots\}$, and let $A$ be a (real) symmetric matrix of rank 2 whose associated quadratic form is positive definite on $\mathbb{N}_{0}$. Then, consider the zeta function of the complex variable $s$ defined by the series of Dirichlet type

$$
\zeta_{2}(s ; A)=\sum_{n \in \mathbb{N}_{0}^{2}}\left(n^{T} A n\right)^{-s},
$$

when $\operatorname{Re}(s)>s_{0}$, for some $s_{0}$ (depending on the matrix $A$ ), and by analytic continuation elsewhere. The aim of this work is to obtain the first coefficients in the Kronecker limit formula for $\zeta_{2}(s ; A)$ near $s=0$. Our result is stated in Theorem 1 (page 197), where we show that the analytic continuation of $\zeta_{2}(s ; A)$ is regular at $s=0$, and we give the values of $\zeta_{2}(0 ; A)$ and $\zeta_{2}^{\prime}(0 ; A)$. The proof of Theorem 1 is based on the more general Spectral Decomposition Lemma (page 188), which provides the first coefficients in the Kronecker limit formula at $s=0$ for the zeta function associated to a more general class of double series of Dirichlet type, whenever some coefficients in the asymptotic expansions of some associated spectral functions are known. We conclude this section with a brief overview on this subject and on the motivations for our analysis.

Let $S=\left\{\lambda_{n}\right\}_{n \in \mathbb{N}_{0}}$ be a sequence of nonvanishing complex numbers with unique accumulation point at infinity and with finite exponent $s_{0}$. Then we define the associated zeta function by the sum

$$
\zeta(s ; S)=\sum_{n=1}^{\infty} \lambda_{n}^{-s},
$$

MSC2000: primary 11R42, 11M36; secondary 11M41.

Keywords: zeta functions, Dirichlet series, Kronecker limit formula. 
for $\operatorname{Re}(s)>s_{0}$, and by analytic extension elsewhere [Atiyah et al. 1973]. Information on the analytic properties of this function, usually called zeta invariants, like poles, residues and particular values, are important in many fields, from number theory to mathematical physics, and different techniques have been defined and applied in different contexts in order to compute such invariants. In particular, we are interested in the value of the derivative of the zeta function at $s=0$, that is, in the coefficient of the linear term in the Kronecker first limit formula at $s=0$ [Weil 1976; Lang 1987]. By a Mellin transform, the zeta function can be immediately associated to a series of theta type $\sum \mathrm{e}^{-\lambda_{n} t}$, and many properties of the zeta function can be easily deduced from the properties of the theta series. Poles, residues and particular values can be obtained whenever the asymptotic expansion of the theta series for small $t$ is available [Gilkey 1995; Rosenberg 1997]; unfortunately, this approach fails when the coefficients of the positive powers of $s$ are involved, as for $\zeta^{\prime}(0 ; S)$. A particularly interesting case is when $\lambda_{n}$ is quadratic in one or more integer variables $\lambda_{n}=n^{T} A n$, where $n$ is an integer vector in $\mathbb{Z}^{k}$, and $A$ a $k$-square real symmetric matrix (zeta functions of this type are called Epstein zeta functions since they were first considered in [Epstein 1903; 1906]). Multidimensional theta series have been deeply studied in the literature. In particular, the Poisson summation formula, namely the Fourier expansion of the theta function, has suitable generalizations to the multidimensional case (see [Chandrasekharan 1985, XI.2, 3], for example). Using these formulas and properties of special functions, it is possible to compute the main zeta invariants for multiple series of Epstein type, also called multiple Eisenstein zeta functions (see [Weil 1976; Ortenzi and Spreafico 2004; Elizalde et al. 1994; Cassou-Noguès 1990] and references therein). Moving up to the zeta functions associated to series of Dirichlet type (namely when the sums are over $\mathbb{N}_{0}^{k}$ ), the main difficulty is precisely the lack of a formula of Poisson type. Consequently, it is hard to find general results, and different techniques have been introduced to deal with the specific cases. See, for example, [Bombieri and Perelli 2001; Carletti and Monti Bragadin 1994a; 1994b; Quine and Choi 1996; Eie 1990; Spreafico 2003; 2004] for simple series or series that can be reduced to simple series. For what concerns multiple series, homogeneous linear series of Dirichlet type, defined (for $\operatorname{Re}(s)>k$ ) by the sum

$$
\zeta(s ; a)=\sum_{n \in \mathbb{N}_{0}^{k}}\left(a_{1} n_{1}+\cdots+a_{k} n_{k}\right)^{-s},
$$

were first introduced by Barnes in his work on multiple gamma functions (see [Cassou-Noguès 1990] for a good review), where he gives formulas for the values at negative integers and relations with the multiple gamma functions that extend the classical Lerch formula (see also [Spreafico 2006]). Notice also the work of Actor [1990], which gives a formula for $\zeta(s ; a)$ as a power series whose coefficients 
are (infinite) sums of Riemann zeta functions and elementary functions and the related works of Matsumoto [1998], where asymptotic expansions are given for nonhomogeneous linear series $\zeta_{1}(s ; a, 1, q)=\sum_{n, k=1}^{\infty}(a n+k+q)^{-s}$, for large $a$, and observe that a formula for $\zeta_{1}^{\prime}(0 ; a, 1)$ was given in [Spreafico 2004], using the Plana Theorem. Next, the case of the double homogeneous quadratic series of Dirichlet type, defined (for $\operatorname{Re}(s)>1$ ) by the sum

$$
\zeta_{2}(s ; a, b, c)=\sum_{n, k=1}^{\infty}\left(a n^{2}+2 c n k+b k^{2}\right)^{-s},
$$

is much harder. These zeta functions (with integer coefficients) appear when dealing with the zeta functions of a narrow ideal class for a real quadratic field, as shown by Zagier [1975; 1977], where he also computes the values at nonpositive integers. The same result was obtained by Shitani [1976] by different methods, while the values at negative half-integers were computed in [Eie 1989]. See also the works of Cassou-Noguès $[1979 ; 1990]$, who, besides the values at negative integers, gave interesting relations with the multiple gamma functions and analyze the $p$-adic analogous of the multiple zeta and gamma functions. In contrast, no results for the derivative $\zeta_{2}^{\prime}(0 ; a, b, c)$ are known, and this is the main motivation for this work.

The technique we develop in order to obtain $\zeta_{2}^{\prime}(0 ; a, b, c)$ is to find a suitable representation of $\zeta_{2}(s ; a, b, c)$, in terms of some complex integral, that allows us to use known information, contained in the asymptotic expansion of some spectral functions associated to $\zeta_{2}(s ; a, b, c)$, to compute $\zeta_{2}^{\prime}(0 ; a, b, c)$ (Theorem 1). This method automatically gives the value of $\zeta_{2}(0 ; a, b, c)$ for all real positive values of the constants $a, b$ and $c$, thus extending one of the results of [Zagier 1977] and also covers the linear case $\zeta_{1}(s ; a, b)$ (see page 197 for details) for all real positive $a$ and $b$. Furthermore, our technique is not defined ad hoc for the case under study in this paper, but can be applied to a larger class of series of Dirichlet type with polynomial general term or even generalized without much effort to series whose general term is not polynomial, and there are works in progress in different directions.

The lemma we are going to prove is a general version of a technique introduced in [Spreafico 2005] to treat the zeta function associated to the Laplacian on a cone. Let $P(x, y)$ be a polynomial in two variables with real coefficients. Consider the double sequence $S=\left\{\lambda_{n, k}=P\left(n^{p}, k^{q}\right)\right\}, n, k \in \mathbb{N}_{0}$, where $p$ and $q$ are some positive real numbers. (For convenience, also assume the numbers $\lambda_{n, k}$ to be ordered in the sequence $\lambda_{1,1} \leq \lambda_{1,2} \leq \lambda_{2,1} \leq \lambda_{1,3} \leq \ldots$.) Assume that $S$ is positive definite (namely $\lambda_{n, k}>0$ for all $n, k$ ), has the unique accumulation point at infinity and is a sequence of finite exponent $s_{0}$; see [Copson 1935, 7.43]. Then there exist finite 
$\alpha$ and $\beta$ such that $\lambda_{n, k}$ behaves like $n^{\alpha}$ for fixed $k$, and like $k^{\beta}$ for fixed $n$, and the series

$$
\zeta(s)=\sum_{n, k=1}^{\infty} \lambda_{n, k}^{-s},
$$

converges absolutely and locally uniformly when $\operatorname{Re}(s)>s_{0}$. In this situation, we can prove the following lemma.

Spectral Decomposition Lemma (SDL). Let $\left\{\lambda_{n, k}\right\}_{n, k \in \mathbb{N}_{0}}$ and $\zeta(s)$ be as above. We have for $\zeta(s)$ the analytic representation

$$
\zeta(s)=\frac{s}{\Gamma(s)} \int_{0}^{\infty} t^{s-1} \frac{1}{2 \pi i} \int_{\Lambda_{\epsilon}} \frac{\mathrm{e}^{-\lambda t}}{-\lambda} T(\lambda, s) d \lambda d t,
$$

where the contour is defined below, and

$$
T(\lambda, s)=\sum_{n=1}^{\infty} n^{-\alpha s} t_{n}(\lambda), \quad t_{n}(\lambda)=-\log \prod_{k=1}^{\infty}\left(1+\frac{n^{\alpha}(-\lambda)}{\lambda_{n, k}}\right) \mathrm{e}^{\sum_{j=1}^{[1 / \beta]} n^{\alpha j} \lambda^{j} /\left(j \lambda_{n, k}^{j}\right)} .
$$

Assume there exist an asymptotic expansion of $t_{n}(\lambda)$ for large $n$, uniformly in $\lambda$ for $\lambda$ in some (unbounded) domain $D$ of the complex plane, disjoint from $S$ and containing the origin $\lambda=0$, and an asymptotic expansion of $t_{n}(\lambda)$, for each fixed $n$, in powers of $-\lambda$ and powers of $-\lambda$ times $\log (-\lambda)$ (like the one considered in [Brüning and Seeley 1987]) for large $\lambda$ in D. Then $\zeta(s)$ can be analytically extended to the whole complex plane up to a set of poles. Moreover, $s=0$ is a regular point and we can compute the main zeta invariants as follows. Let $f(\lambda)$ be the coefficient of the $1 / n$ term in the asymptotic expansion of $t_{n}(\lambda)$ for large $n$, and define

Then

$$
p_{n}(\lambda)=t_{n}(\lambda)-\frac{1}{n} f(\lambda), \quad P(\lambda, s)=\sum_{n=1}^{\infty} n^{-\alpha s} p_{n}(\lambda)
$$

$$
\begin{aligned}
& \zeta(0)=-A(0)+\frac{1}{\alpha} \operatorname{Res}_{1}(F(s), s=0), \\
& \zeta^{\prime}(0)=-A^{\prime}(0)-B(0)+\frac{1}{\alpha} \operatorname{Res}_{0}(F(s), s=0)+\gamma\left(1+\frac{1}{\alpha}\right) \operatorname{Res}_{1}(F(s), s=0),
\end{aligned}
$$

where

$$
F(s)=\int_{0}^{\infty} t^{s-1} \frac{1}{2 \pi i} \int_{\Lambda_{\epsilon}} \frac{\mathrm{e}^{-\lambda t}}{-\lambda} f(\lambda) d \lambda d t
$$

and

$$
A(s)=\sum_{n=1}^{\infty} n^{-\alpha s} a_{n}, \quad B(s)=\sum_{n=1}^{\infty} n^{-\alpha s} b_{n},
$$

$a_{n}$ and $b_{n}$ being the logarithmic and constant terms in the asymptotic expansion of $p_{n}(\lambda)$ for large $\lambda$ and fixed $n$. 
Proof. The general plan is as follows. We know how to deal with a simple series [Spreafico 2006], say the sum over $k$, and we can rearrange the sums so as to decompose the double series as a sum over $n$ of sums over $k$. The problem in this decomposition is that we have to regularize also the external sum in $n$. For that purpose, it is enough to collect an opportune power of $n$. This will give a suitable analytic representation and will keep track of all contributions in the regularization process.

As a first step, we provide an analytic representation for $\zeta(s)$. Notice that all the series and the products appearing in the following calculations converge absolutely and uniformly, due to the assumptions on the sequence $\left\{\lambda_{n, k}\right\}$. We can write

$$
\begin{aligned}
\zeta(s) & =\sum_{n=1}^{\infty} n^{-\alpha s} \sum_{k=1}^{\infty}\left(\frac{\lambda_{n, k}}{n^{\alpha}}\right)^{-s}=\frac{1}{\Gamma(s)} \int_{0}^{\infty} t^{s-1} \sum_{n=1}^{\infty} n^{-\alpha s} \sum_{k=1}^{\infty} \mathrm{e}^{-\frac{\lambda_{n, k}}{n^{\alpha}} t} d t \\
& =\frac{1}{\Gamma(s)} \int_{0}^{\infty} t^{s-1} \sum_{n=1}^{\infty} n^{-\alpha s} \frac{1}{2 \pi i} \int_{\Lambda_{\epsilon}} \mathrm{e}^{-\lambda t} \sum_{k=1}^{\infty} \frac{1}{\lambda-\lambda_{n, k} / n^{\alpha}} d \lambda d t,
\end{aligned}
$$

where $\Lambda_{\epsilon}=\partial D$ can be deformed to the contour $\{\lambda \in \mathbb{C}|| \arg (\lambda-\epsilon) \mid=\theta / 2\}$, oriented counterclockwise, with some nonnegative real $\epsilon<\lambda_{1,1}$ and $0<\theta<\pi$. The preceding expression in turn equals

$$
\frac{1}{\Gamma(s)} \int_{0}^{\infty} t^{s-1} \frac{1}{2 \pi i} \int_{\Lambda_{\epsilon}} \mathrm{e}^{-\lambda t} R(\lambda, s) d \lambda d t
$$

where

$$
R(\lambda, s)=\sum_{n=1}^{\infty} n^{-\alpha s} r_{n}(\lambda), \quad r_{n}(\lambda)=-\sum_{k=1}^{\infty}\left(\frac{n^{\alpha}}{n^{\alpha}(-\lambda)+\lambda_{n, k}}-\sum_{j=0}^{[1 / \beta]-1} \frac{n^{j \alpha} \lambda^{j}}{\lambda_{n, k}^{j+1}}\right) .
$$

We can now introduce a primitive function for the resolvent $R(\lambda, s)$; namely, let

$$
T(\lambda, s)=\sum_{n=1}^{\infty} n^{-\alpha s} t_{n}(\lambda), \quad t_{n}(\lambda)=-\log \prod_{k=1}^{\infty}\left(1+\frac{n^{\alpha}(-\lambda)}{\lambda_{n, k}}\right) \mathrm{e}^{\sum_{j=1}^{[1 / \beta]} n^{\alpha j} \lambda^{j} /\left(j \lambda_{n, k}^{j}\right)} .
$$

Then

$$
-\frac{d}{d \lambda} T(\lambda, s)=R(\lambda, s),
$$

and again the canonical product converges; moreover, the exponential factor makes no contribution to the quantities we are going to compute, since it adds terms to $R(\lambda, s)$ that vanish after integration over $\lambda$ (see [Spreafico 2005]). Also notice that, since $T(\lambda, s)$ is defined up to a constant, we can always chose it so that $T(0, s)=0$, 
and we do so. Integrating by parts first in $\lambda$ and then in $t$ we get

$$
\zeta(s)=\frac{s}{\Gamma(s)} \int_{0}^{\infty} t^{s-1} \frac{1}{2 \pi i} \int_{\Lambda_{\epsilon}} \frac{\mathrm{e}^{-\lambda t}}{-\lambda} T(\lambda, s) d \lambda d t .
$$

We could now use this representation to compute both the value at $s=0$ and the derivative, if we could provide an expansion for $T(\lambda, s)$ for large $\lambda$ [Spreafico 2004]. The problem is that more care is necessary due to the possible appearance of a singularity in the sum over $n$. The unique possible pole in such a sum comes from a term behaving like $1 / n$ in the expansion of $t_{n}$ for large $n$ uniformly in $\lambda$, so we can overcome this problem as follows. Let $f(\lambda)$ be the coefficient of the term in $1 / n$ in the uniform expansion of $t_{n}(\lambda)$ for large $n$, and define

$$
p_{n}(\lambda)=t_{n}(\lambda)-\frac{1}{n} f(\lambda), \quad P(\lambda, s)=\sum_{n=1}^{\infty} n^{-\alpha s} p_{n}(\lambda) .
$$

Then

$$
\begin{aligned}
\zeta(s)=\frac{s}{\Gamma(s)} \int_{0}^{\infty} t^{s-1} \frac{1}{2 \pi i} \int_{\Lambda_{\epsilon}} \frac{\mathrm{e}^{-\lambda t}}{-\lambda} P(\lambda, s) d \lambda d t \\
\quad+\frac{s}{\Gamma(s)} \zeta_{R}(\alpha s+1) \int_{0}^{\infty} t^{s-1} \frac{1}{2 \pi i} \int_{\Lambda_{\epsilon}} \frac{\mathrm{e}^{-\lambda t}}{-\lambda} f(\lambda) d \lambda d t \\
=z_{1}(s)+z_{2}(s),
\end{aligned}
$$

and the singular contribution is isolated in the second term. We can deal with the two terms as follows. For the first, the assumed expansion of $t_{n}(\lambda)$ for large $\lambda$ in $D$ and fixed $n$, gives the following expansion of $p_{n}(\lambda)$,

$p_{n}(\lambda)=t_{n}(\lambda)-\frac{1}{n} f(\lambda)=\sum_{\gamma} c_{\gamma, n}(-\lambda)^{\gamma_{0}-\gamma}+\sum_{\delta} d_{\delta, n}(-\lambda)^{\delta_{0}-\delta} \log (-\lambda)+u_{n}(\lambda)$,

where $\gamma$ and $\delta$ run through some finite sets of real positive numbers with $\gamma-\gamma_{0}$, $\delta-\delta_{0}<M$, for some positive $M, \gamma_{0}$ and $\delta_{0}$ [Brüning and Seeley 1987], and the reminder is such that for $\lambda \in D$,

$$
\lim _{\lambda \rightarrow \infty} \frac{u_{n}(\lambda)}{(-\lambda)^{-M}}=0 .
$$

Moreover, because the expansion of $t_{n}(\lambda)$ is uniform for large $n$, we know that the coefficients $c_{\gamma, n}, d_{\delta, n}$ and the function $u_{n}(\lambda)$ have an expansion for large $n$ without terms of the form $1 / n$ and such that the series

$$
\sum_{n=1}^{\infty} c_{\gamma, n} n^{-\alpha s}, \quad \sum_{n=1}^{\infty} d_{\delta, n} n^{-\alpha s}, \quad \sum_{n=1}^{\infty} u_{n}(\lambda) n^{-\alpha s}
$$


converge uniformly for $\operatorname{Re}(s)>s_{0}$ and $\lambda \in D$, and have analytic extensions regular at $s=0$. This means that we can use classical methods to deal with the analytic extension of functional zeta functions (see [Gilkey 1995] or [Rosenberg 1997]). In particular we need the constant and logarithmic terms. Hence let's write

$$
p_{n}(\lambda)=\cdots+a_{n} \log (-\lambda)+b_{n}+\cdots,
$$

thus obtaining

$$
P(\lambda, s)=\cdots+A(s) \log (-\lambda)+B(s)+\cdots,
$$

with

$$
A(s)=\sum_{n=1}^{\infty} n^{-\alpha s} a_{n}, \quad B(s)=\sum_{n=1}^{\infty} n^{-\alpha s} b_{n} .
$$

Following the standard approach (see for example [Gilkey 1995]), we split the $t$ integral at $t=1$. The $t>1$ part defines a regular function of $s$ near $s=0$, while in the $t<1$ part we must change the contour of the $\lambda$ integral (here $C_{\epsilon}$ is a circle around the origin of ray $\epsilon$ ) and then we can rescale $\lambda$ by $t$ and use the expansion above to obtain (see [Spreafico 2006])

$z_{1}(s)$

$$
\begin{array}{r}
=\frac{s}{\Gamma(s)} \int_{0}^{1} t^{s-1}\left(\frac{1}{2 \pi i} \int_{\Lambda_{-\epsilon}} \frac{\mathrm{e}^{-\lambda}}{-\lambda} P(\lambda / t, s) d \lambda-\frac{1}{2 \pi i} \int_{C_{\epsilon}} \frac{\mathrm{e}^{-\lambda t}}{-\lambda} P(\lambda, s) d \lambda\right) d t \\
+s^{2} h_{1}(s) \\
=\frac{s}{\Gamma(s)} \int_{0}^{1} t^{s-1}\left(\frac{1}{2 \pi i} \int_{\Lambda_{-\epsilon}} \frac{\mathrm{e}^{-\lambda}}{-\lambda}\left(A(s) \log \frac{-\lambda}{t}+B(s)\right) d \lambda+P(0, s)\right) d t \\
+s^{2} h_{2}(s)
\end{array}
$$

where the $h_{i}(s)$ are regular functions of $s$ near $s=0$. At this point notice that $P(0, s)=0$ for all $s$, since $\lambda=0$ is assumed to belong to the domain $D$. After some computations we obtain

$$
z_{1}(s)=\frac{s}{\Gamma(s+1)}\left(\gamma A(s)-\frac{A(s)}{s}-B(s)\right)+s^{2} h_{2}(s) .
$$

This expression allows us to deal with both $\zeta(0)$ and $\zeta^{\prime}(0)$. In fact,

$$
z_{1}(0)=-A(0)
$$

while, by taking the derivative near $s=0$ and using the known expansion for the inverse of the gamma function, we obtain

$$
z_{1}^{\prime}(0)=-A^{\prime}(0)-B(0)
$$


The second term, $z_{2}(s)$, can be treated by using the expansions for the different factors as functions of $s$. Writing

$$
z_{2}(s)=\frac{s}{\Gamma(s)} \zeta_{R}(\alpha s+1) F(s)
$$

with

$$
F(s)=\int_{0}^{\infty} t^{s-1} \frac{1}{2 \pi i} \int_{\Lambda_{\epsilon}} \frac{\mathrm{e}^{-\lambda t}}{-\lambda} f(\lambda) d \lambda d t
$$

we obtain

$$
z_{2}(s)=\frac{F_{1}}{\alpha}+\frac{1}{\alpha}\left(F_{0}+(\alpha+1) \gamma F_{1}\right) s+O\left(s^{2}\right),
$$

and hence

$$
z_{2}(0)=\frac{1}{\alpha} \operatorname{Res}_{1}(F(s), s=0),
$$

and

$$
z_{2}^{\prime}(0)=\frac{1}{\alpha}\left(\operatorname{Res}_{0}(F(s), s=0)+\gamma(\alpha+1) \operatorname{Res}_{1}(F(s), s=0)\right) .
$$

The Spectral Decomposition Lemma is a theoretical result that becomes effective when a concrete expansion for $t_{n}(\lambda)$ is available; this is actually the case in many interesting examples, such as the ones we will deal with in the next sections or the one studied in [Spreafico 2005].

\section{The homogeneous quadratic double case}

In this section, we consider the quadratic case, namely the zeta function defined by the series

$$
\zeta_{2}(s ; a, b, c)=\sum_{n \in \mathbb{N}_{0}^{2}}\left(n^{T} A n\right)^{-s}=\sum_{n, k=1}^{\infty}\left(a n^{2}+b k^{2}+2 c n k\right)^{-s},
$$

when $\operatorname{Re}(s)>1$, and by analytic continuation elsewhere. Here $A=\left(\begin{array}{ll}a & c \\ c & b\end{array}\right)$ is a real symmetric matrix with positive definite associated quadratic form on $\mathbb{N}_{0}$. We introduce the notation $\Delta=c^{2}-a b=-\operatorname{det} A$, and we further assume $a b \neq 0$. To compute the zeta invariants at $s=0$ we apply the SDL. Since $a b \neq 0$, we have $\alpha=\beta=2$, and

$$
\zeta_{2}(s ; a, b, c)=\frac{s^{2}}{\Gamma(s+1)} \int_{0}^{\infty} t^{s-1} \frac{1}{2 \pi i} \int_{\Lambda_{\epsilon}} \frac{\mathrm{e}^{-\lambda t}}{-\lambda} T(\lambda, s) d \lambda,
$$

where

$$
T(\lambda, s)=\sum_{n=1}^{\infty} n^{-2 s} t_{n}(\lambda), \quad t_{n}(\lambda)=-\log \prod_{k=1}^{\infty}\left(1+\frac{n^{2}(-\lambda)}{a n^{2}+2 c n k+b k^{2}}\right) .
$$


Since $a n^{2}+2 c n k+b k^{2}=b(k+c n / b)^{2}-\Delta n^{2} / b$, we obtain

$$
t_{n}(\lambda)=-\log \prod_{k=1}^{\infty}\left(1+\frac{-\lambda n^{2} / b-\Delta n^{2} / b^{2}}{(k+c n / b)^{2}}\right)+\log \prod_{k=1}^{\infty}\left(1+\frac{-\Delta n^{2} / b^{2}}{(k+c n / b)^{2}}\right) .
$$

Using the product definition of the gamma function we get

$$
\begin{array}{r}
t_{n}(\lambda)=\log \Gamma\left(\frac{c+\sqrt{\Delta-b(-\lambda)}}{b} n\right)+\log \Gamma\left(\frac{c-\sqrt{\Delta-b(-\lambda)}}{b} n\right) \\
\quad-\log \Gamma\left(\frac{c+\sqrt{\Delta}}{b} n\right)-\log \Gamma\left(\frac{c-\sqrt{\Delta}}{b} n\right)+\log \left(1+\frac{-\lambda}{a}\right) .
\end{array}
$$

The expansion for large $n$ is

$$
\begin{aligned}
\log & \Gamma((x+y) n)+\log \Gamma((x-y) n) \\
= & \left((x+y) n-\frac{1}{2}\right) \log (x+y) n-(x+y) n+\frac{1}{2} \log 2 \pi+\frac{1}{12(x+y) n} \\
& \quad+\left((x-y) n-\frac{1}{2}\right) \log (x-y) n-(x-y) n+\frac{1}{2} \log 2 \pi+\frac{1}{12(x-y) n}+O\left(n^{-3}\right) \\
& =\cdots+\frac{1}{6\left(x^{2}-y^{2}\right) n}+\cdots,
\end{aligned}
$$

with $x=c / b$ and $y_{\lambda}=\sqrt{\Delta-b(-\lambda)} / b$. Notice that $\lambda=0$ belongs to the domain where the expansion is uniform. The expansion of the whole $t_{n}(\lambda)$ will be

$$
t_{n}(\lambda)=\cdots+\frac{x}{6\left(x^{2}-y_{\lambda}^{2}\right)}-\frac{x}{6\left(x^{2}-y_{0}^{2}\right)}+\cdots,
$$

which gives

$$
f(\lambda)=\frac{c}{6}\left(\frac{1}{a-\lambda}-\frac{1}{a}\right) .
$$

Therefore,

$$
\begin{aligned}
F(s) & =\frac{c}{6} \int_{0}^{\infty} t^{s-1} \frac{1}{2 \pi i} \int_{\Lambda_{\epsilon}} \frac{\mathrm{e}^{-\lambda t}}{-\lambda}\left(\frac{1}{a-\lambda}-\frac{1}{a}\right) d \lambda d t \\
& =\frac{c}{6 a} a^{-s} \int_{0}^{\infty} t^{s-1} \frac{1}{2 \pi i} \int_{\Lambda_{\epsilon}} \frac{\mathrm{e}^{-\lambda t}}{-\lambda}\left(\frac{1}{1-\lambda}-1\right) d \lambda d t \\
& =\frac{c}{6 a} a^{-s} \int_{0}^{\infty} t^{s-1} \Gamma(1, t) d t=\frac{c}{6 a} a^{-s} \Gamma(s),
\end{aligned}
$$

where we have used the definition of the incomplete gamma function to compute the last integral and get the last equality. Notice that in the integral defining $F(s)$, the constant term gives no contribution and $F(s)$ is not regular at $s=0$. Expanding 
we obtain

$$
F(s)=\frac{c}{6 a}\left(\frac{1}{s}-\gamma-\log a+O(s)\right)
$$

and hence

$$
\operatorname{Res}_{0}(F(s), s=0)=-\frac{c}{6 a}(\gamma+\log a), \quad \operatorname{Res}_{1}(F(s), s=0)=\frac{c}{6 a} .
$$

Next, we compute the expansion of $p_{n}(\lambda)$ for large $\lambda$ :

$$
\begin{aligned}
t_{n}(\lambda)=\left(x n-\frac{1}{2}\right) & \log \left(x^{2}-y_{\lambda}^{2}\right) n^{2}+n y_{\lambda} \log \frac{x+y_{\lambda}}{x-y_{\lambda}}+\log 2 \pi-2 x n \\
& -\log \Gamma\left(\left(x+y_{0}\right) n\right) \Gamma\left(\left(x-y_{0}\right) n\right)+\log (-\lambda)-\log a+O\left((-\lambda)^{-1}\right),
\end{aligned}
$$

with $y_{\lambda}=\sqrt{\Delta-b(-\lambda)} / b$. Setting $y_{\lambda}=i u_{\lambda}, u_{\lambda}=\sqrt{b(-\lambda)-\Delta} / b \sim \sqrt{-\lambda}$, we can expand

$$
\begin{aligned}
n y_{\lambda} \log \frac{x+y_{\lambda}}{x-y_{\lambda}} & =i n u_{\lambda} \log \frac{x+i u_{\lambda}}{x-i u_{\lambda}}=-2 n u_{\lambda} \arctan \frac{u_{\lambda}}{x} n \\
& =-\pi n u_{\lambda}+2 x n+O\left(u_{\lambda}^{-2}\right)=-\pi n \sqrt{-\lambda}+2 x n+O\left((-\lambda)^{-1}\right), \\
\log \left(x^{2}-y_{\lambda}^{2}\right) n^{2} & =\log (-\lambda)+\log \frac{n^{2}}{b}+O\left((-\lambda)^{-1}\right), \\
f(\lambda) & =-\frac{c}{6 a}+O\left((-\lambda)^{-1}\right)
\end{aligned}
$$

to obtain

$$
\begin{array}{r}
p_{n}(\lambda)=\left(x n-\frac{1}{2}\right)\left(\log (-\lambda)+\log \frac{n^{2}}{b}\right)-\pi n \sqrt{-\lambda}+\log (-\lambda)-\log a+\log 2 \pi \\
-\log \Gamma\left(\left(x+y_{0}\right) n\right) \Gamma\left(\left(x-y_{0}\right) n\right)+\frac{c}{6 a n}+O\left((-\lambda)^{-1}\right) .
\end{array}
$$

This means that

$$
\begin{aligned}
a_{n} & =\frac{c}{b} n+\frac{1}{2}, \\
b_{n}=-\log \Gamma\left(\frac{c+\sqrt{\Delta}}{b} n\right) \Gamma\left(\frac{c-\sqrt{\Delta}}{b} n\right) & \quad+\log 2 \pi+\left(\frac{c}{b} n-\frac{1}{2}\right) \log \frac{n^{2}}{b}-\log a+\frac{c}{6 a n},
\end{aligned}
$$

and gives

$$
\begin{gathered}
A(s)=\sum_{n=1}^{\infty} a_{n} n^{-\alpha s}=\frac{c}{b} \zeta_{R}(2 s-1)+\frac{1}{2} \zeta_{R}(2 s), \\
A(0)=-\frac{c}{12 b}-\frac{1}{4}, \quad A^{\prime}(0)=-\frac{1}{2} \log 2 \pi+\frac{2 c}{b} \zeta_{R}^{\prime}(-1) .
\end{gathered}
$$


We have obtained

$$
\zeta_{2}(0 ; a, b, c)=\frac{1}{4}+\frac{c}{12 a}+\frac{c}{12 b} .
$$

Next

$$
\begin{aligned}
B(s)=\sum_{n=1}^{\infty} n^{-2 s} b_{n}=\sum_{n=1}^{\infty}(- & \log \Gamma\left(\frac{c+\sqrt{\Delta}}{b} n\right) \Gamma\left(\frac{c-\sqrt{\Delta}}{b} n\right) \\
& \left.+\log 2 \pi-\log a+\left(\frac{c}{b} n-\frac{1}{2}\right) \log \frac{n^{2}}{b}+\frac{c}{6 a n}\right) n^{-2 s} .
\end{aligned}
$$

By the SDL (page 188), $B(s)$ has an analytic extension regular at $s=0$. We provide two different representations. Let

$$
\begin{aligned}
\log \Gamma\left(\frac{c+\sqrt{\Delta}}{b} n\right) \Gamma\left(\frac{c-\sqrt{\Delta}}{b} n\right) \\
=\left(\frac{c}{b} n-\frac{1}{2}\right) \log \frac{a n^{2}}{b}+\frac{\sqrt{\Delta}}{b} n \log \frac{c+\sqrt{\Delta}}{c-\sqrt{\Delta}}+\log 2 \pi-\frac{2 c n}{b}+K,
\end{aligned}
$$

where

(1)

$$
K=\frac{1}{2} \sum_{m=1}^{\infty} \frac{m}{(m+1)(m+2)} \sum_{k=1}^{\infty}\left(\frac{1}{(k+(c+\sqrt{\Delta}) n / b)^{m+1}}+\frac{1}{(k+(c-\sqrt{\Delta}) n / b)^{m+1}}\right)
$$

or

$$
K=2 \int_{0}^{\infty}\left(\arctan \frac{b t}{(c+\sqrt{\Delta}) n}+\arctan \frac{b t}{(c-\sqrt{\Delta}) n}\right) \frac{d t}{\mathrm{e}^{2 \pi t}-1} .
$$

The former gives

$$
\begin{aligned}
& B(s)=\left(\frac{2 c}{b}-\frac{c}{b} \log a-\frac{\sqrt{\Delta}}{b} \log \frac{c+\sqrt{\Delta}}{c-\sqrt{\Delta}}\right) \zeta_{R}(2 s-1)-\frac{1}{2} \log a \zeta_{R}(2 s) \\
& -\frac{1}{2} \sum_{n=1}^{\infty} n^{-2 s}\left(\sum_{m=1}^{\infty} \frac{m\left(\zeta_{H}(m+1,(c+\sqrt{\Delta}) n / b)+\zeta_{H}(m+1,(c-\sqrt{\Delta}) n / b)\right)}{(m+1)(m+2)}-\frac{c}{3 a n}\right) .
\end{aligned}
$$

Thus,

$$
\begin{aligned}
B(0) & =-\frac{1}{12}\left(\frac{2 c}{b}-\frac{c}{b} \log a-\frac{\sqrt{\Delta}}{b} \log \frac{c+\sqrt{\Delta}}{c-\sqrt{\Delta}}\right)+\frac{1}{4} \log a \\
- & \frac{1}{2} \sum_{n=1}^{\infty}\left(\sum_{m=1}^{\infty} \frac{m\left(\zeta_{H}(m+1,(c+\sqrt{\Delta}) n / b)+\zeta_{H}(m+1,(c-\sqrt{\Delta}) n / b)\right)}{(m+1)(m+2)}-\frac{c}{3 a n}\right)
\end{aligned}
$$


and

$$
\begin{aligned}
& \zeta^{\prime}(0 ; a, b, c) \\
& =\frac{1}{2} \log 2 \pi-\left(\frac{1}{4}+\frac{c}{12 a}+\frac{c}{12 b}\right) \log a-\frac{\sqrt{\Delta}}{12 b} \log \frac{c+\sqrt{\Delta}}{c-\sqrt{\Delta}}+\frac{c}{6 b}+\frac{\gamma c}{6 a}-\frac{2 c}{b} \zeta_{R}^{\prime}(-1) \\
& \quad+\frac{1}{2} \sum_{n=1}^{\infty}\left(\sum_{m=1}^{\infty} \frac{m\left(\zeta_{H}(m+1,(c+\sqrt{\Delta}) n / b)+\zeta_{H}(m+1,(c-\sqrt{\Delta}) n / b)\right)}{(m+1)(m+2)}-\frac{c}{3 a n}\right) .
\end{aligned}
$$

Computing the limit of these expressions as $c \rightarrow 0$ is not easy.

Using the second representation for $K$, equation (2), and assuming $a, b$ and $c$ positive we obtain

$$
K=2 \int_{0}^{\infty} \arctan \frac{2 c n t}{a n^{2}-b t^{2}} \frac{d t}{\mathrm{e}^{2 \pi t}-1}-\log \left(1-\mathrm{e}^{-2 \pi \sqrt{a / b} n}\right) .
$$

Notice that this holds for real or pure imaginary $\sqrt{\Delta}$, and that the assumption that $a, b, c$ are positive is not necessary. In general, the sign of $\operatorname{Re}(z) /(1+\operatorname{Im}(z))$, where $z=b /(c+\sqrt{\Delta})$, will give the sign of the term $-\log \left(1-\mathrm{e}^{-2 \pi \sqrt{a / b} n}\right)$.

Formula (3) can be used to show that the sum appearing in $\zeta_{2}^{\prime}(0 ; a, b, c)$ is regular at $s=0$, and to deal with the case $c=0$, as will be done in the last section. For the first point, consider that, for large $n$,

$$
\int_{0}^{\infty} \arctan \frac{2 c t n}{a n^{2}-b t^{2}} \frac{d t}{\mathrm{e}^{2 \pi t}-1}=\frac{c}{12 a n}+O\left(n^{-3}\right) .
$$

The complete formula for the derivative with the representation for $K$ given in (3) is stated in the next theorem, which generalizes the Kronecker first limit formula to the function $\zeta_{2}(s ; A)$.

We let

$$
\eta(z)=\mathrm{e}^{(\pi / 12) i z} \prod_{n=1}^{\infty}\left(1-\mathrm{e}^{2 \pi i n z}\right)
$$

be the Dedekind eta function [Chandrasekharan 1985].

Theorem 1. The function $\zeta_{2}(s ; A)$ has near $s=0$ the expansion

$$
\zeta_{2}(s ; A)=\zeta_{2}(0 ; A)+\zeta_{2}^{\prime}(0 ; A) s+O\left(s^{2}\right),
$$

where $A=\left(\begin{array}{ll}a & c \\ c & b\end{array}\right)$, with real $a, b, c>0, \Delta=c^{2}-a b=-\operatorname{det} A$,

$$
\zeta_{2}(0 ; A)=\frac{1}{4}+\frac{c}{12 a}+\frac{c}{12 b},
$$




$$
\begin{aligned}
& \zeta_{2}^{\prime}(0 ; A)=\frac{1}{2} \log 2 \pi-\left(\frac{1}{4}+\frac{c}{12 a}+\frac{c}{12 b}\right) \log a-\frac{\sqrt{\Delta}}{12 b} \log \frac{c+\sqrt{\Delta}}{c-\sqrt{\Delta}} \\
&+\frac{c}{6 b}+\frac{\gamma c}{6 a}-\frac{2 c}{b} \zeta_{R}^{\prime}(-1)-\frac{\pi}{12} \sqrt{\frac{a}{b}}-\log \eta\left(i \sqrt{\frac{a}{b}}\right) \\
&+2 \sum_{n=1}^{\infty}\left(\int_{0}^{\infty} \arctan \frac{2 c n t}{a n^{2}-b t^{2}} \frac{d t}{\mathrm{e}^{2 \pi t}-1}-\frac{c}{12 a n}\right) .
\end{aligned}
$$

The value of $\zeta_{2}(0 ; A)$ agrees with the one given in [Zagier 1977, Section 2] for the case $\Delta>0$.

In the theorem, the positivity of $a, b$ and $c$ need not be assumed if we use the representation of $K$ given in formula (1), but then we need to assume that the quadratic from associated to the matrix $A$ is positive definite on $\mathbb{N}_{0}$.

\section{Particular cases}

Case $\boldsymbol{c}=\mathbf{0}$. The case $c=0$ can be dealt with directly by using the Kronecker first limit formula [Lang 1987; Ortenzi and Spreafico 2004] or by taking the limit of the result obtained in Section 2, and this shows that Theorem 1 reduces continuously to the classical Kronecker formula. We get

$$
\begin{aligned}
\zeta_{2}(0 ; a, b, 0) & =\frac{1}{4}, \\
\zeta_{2}^{\prime}(0 ; a, b, 0) & =\frac{1}{2} \log 2 \pi-\frac{1}{4} \log a-\log \eta\left(i \sqrt{\frac{a}{b}}\right) \\
& =\frac{1}{2} \log 2 \pi-\frac{1}{4} \log a+\frac{\pi}{12} \sqrt{\frac{a}{b}}-\log \prod_{n=1}^{\infty}\left(1-\mathrm{e}^{-2 \pi \sqrt{a / b} n}\right) .
\end{aligned}
$$

Case $\Delta=0$ and linear case. When $\Delta=0$, that is, $c=\sqrt{a b}$, we have $a n^{2}+2 c n k+$ $b k^{2}=(\sqrt{a} n+\sqrt{b} k)^{2}$, and thus we reduce to the linear case, namely

$$
\zeta_{2}(s ; a, b, \sqrt{a b})=\zeta_{1}(2 s ; \sqrt{a}, \sqrt{b}),
$$

where $\zeta_{1}(s ; a, b)$ is defined for real positive $a$ and $b$ by the sum

$$
\zeta_{1}(s ; a, b)=\sum_{n, k=1}^{\infty}(a n+b k)^{-s}
$$

when $\operatorname{Re}(s)>2$, and by analytic continuation elsewhere. For completeness we give the values of $\zeta_{1}(0 ; a, b)$ and $\zeta_{1}^{\prime}(0 ; a, b)$ that can be obtained from Theorem 1 or by direct application of the SDL (page 188). We give two equivalent formulas for the derivative.

$$
\zeta_{1}(0 ; a, b)=\frac{1}{4}+\frac{a}{12 b}+\frac{b}{12 a}
$$




$$
\begin{aligned}
\zeta_{1}^{\prime}(0 ; a, b)=\frac{b}{12 a}(\gamma- & \log a)+\frac{a}{12 b}(1-\log a)+\frac{1}{4}(\log 2 \pi-\log a)-\frac{a}{b} \zeta_{R}^{\prime}(-1) \\
+ & \frac{1}{2} \sum_{n=1}^{\infty}\left(\sum_{m=1}^{\infty} \frac{m}{(m+1)(m+2)} \zeta_{H}\left(m+1, \frac{a}{b} n+1\right)-\frac{b}{6 a n}\right) \\
=\frac{b}{12 a}(\gamma-\log a)+\frac{a}{12 b}(1-\log a)+\frac{1}{4}(\log 2 \pi-\log a)-\frac{a}{b} \zeta_{R}^{\prime}(-1) & +2 \sum_{n=1}^{\infty}\left(\sum_{m=1}^{\infty} \int_{0}^{\infty} \arctan \frac{b t}{a n} \frac{d t}{\mathrm{e}^{2 \pi t}-1}-\frac{b}{24 a n}\right) .
\end{aligned}
$$

The result for $\zeta_{1}(0 ; a, b)$ is consistent with the one obtained by using the formula in [Cassou-Noguès 1990, Section 4], and if, say, $a=1$ and $0<b<2$, then also with the one computed by applying [Actor 1990, (19)]. As to the derivative, it seems that a direct application of Actor's formula has problems of convergence, and one must use equation (8) of the same paper instead.

Case $a=0$. The semilinear case $a=0$ does not reduce to any other one, and in fact it can be checked that we have a pole at $s=\frac{3}{2}$. Beside, applying the SDL we find out that the behavior at $s=0$ reduces to the behavior of an appropriate linear case. We have $\alpha=2, \beta=1$, and we can choose to collect $n$ or $k$ when applying the SDL. In the two cases, we get

$$
\begin{aligned}
& t_{n}(\lambda)=-\log \prod_{k=1}^{\infty}\left(1+\frac{n(-\lambda)}{2 c n k+b k^{2}}\right), \\
& t_{k}(\lambda)=-\log \prod_{n=1}^{\infty}\left(1+\frac{k(-\lambda)}{2 c n+b k}\right) .
\end{aligned}
$$

Using the first of these formulas, we find out that the domain of validity of the asymptotic expansion for large $n$ does not contain the origin $\lambda=0$, and hence we cannot apply the SDL properly. Using the second expression, we see that this is the same function appearing in the linear case of $\zeta_{1}(s ; b, 2 c)$, and hence

$$
\begin{aligned}
& \zeta_{2}(0 ; 0, b, c)=\zeta_{1}(0 ; b, 2 c), \\
& \zeta_{2}^{\prime}(0 ; 0, b, c)=\zeta_{1}^{\prime}(0 ; b, 2 c) .
\end{aligned}
$$

\section{Acknowledgment}

The author is deeply indebted to the referee for his most valuable remarks, which helped fashion the present form of this work, and for suggesting many very useful references. 


\section{References}

[Actor 1990] A. Actor, "Evaluation of multidimensional linear zeta functions", J. Number Theory 35:1 (1990), 62-71. MR 91d:11108 Zbl 0703.11038

[Atiyah et al. 1973] M. Atiyah, R. Bott, and V. K. Patodi, "On the heat equation and the index theorem”, Invent. Math. 19 (1973), 279-330. MR 58 \#31287 Zbl 0257.58008

[Bombieri and Perelli 2001] E. Bombieri and A. Perelli, "Zeros and poles of Dirichlet series", Atti Accad. Naz. Lincei Cl. Sci. Fis. Mat. Natur. Rend. Lincei (9) Mat. Appl. 12 (2001), 69-73 (2002). MR 1898450 (2003i:30002) Zbl 02216703

[Brüning and Seeley 1987] J. Brüning and R. Seeley, "The resolvent expansion for second order regular singular operators”, J. Funct. Anal. 73:2 (1987), 369-429. MR 88g:35151 Zbl 0625.47040

[Carletti and Monti Bragadin 1994a] E. Carletti and G. Monti Bragadin, "On Dirichlet series associated with polynomials”, Proc. Amer. Math. Soc. 121:1 (1994), 33-37. MR 1179586 (94g:11066) Zbl 0804.11051

[Carletti and Monti Bragadin 1994b] E. Carletti and G. Monti Bragadin, "On MinakshisundaramPleijel zeta functions of spheres”, Proc. Amer. Math. Soc. 122:4 (1994), 993-1001. MR 95b:58164 Zbl 0838.11057

[Cassou-Noguès 1979] P. Cassou-Noguès, "Valeurs aux entiers négatifs des fonctions zêta et fonctions zêta p-adiques", Invent. Math. 51:1 (1979), 29-59. MR 80h:12009b Zbl 0408.12015

[Cassou-Noguès 1990] P. Cassou-Noguès, "Dirichlet series associated with a polynomial”, pp. 244252 in Number theory and physics (Les Houches, 1989), edited by J. M. Luck et al., Springer Proc. Phys. 47, Springer, Berlin, 1990. MR 1058469 (91e:11097) Zbl 0713.11063

[Chandrasekharan 1985] K. Chandrasekharan, Elliptic functions, Grundlehren der Math. Wiss. 281, Springer, Berlin, 1985. MR 87e:11058 Zbl 0575.33001

[Copson 1935] E. T. Copson, An introduction to the theory of functions of a complex variable, Clarendon, Oxford, 1935. Zbl 0012.16902

[Eie 1989] M. K. Eie, "On the values at negative half-integers of the Dedekind zeta function of a real quadratic field”, Proc. Amer. Math. Soc. 105:2 (1989), 273-280. MR 90a:11137 Zbl 0667.10013

[Eie 1990] M. K. Eie, "On a Dirichlet series associated with a polynomial”, Proc. Amer. Math. Soc. 110:3 (1990), 583-590. MR 91m:11071 Zbl 0708.11040

[Elizalde et al. 1994] E. Elizalde, S. Odintsov, A. Romeo, A. A. Bytsenko, and S. Zerbini, Zeta regularization techniques with applications, World Scientific, River Edge, NJ, 1994. MR 96m:81156 Zbl 1050.81500

[Epstein 1903] P. Epstein, “Zur Theorie allgemeiner Zetafunctionen”, Math. Ann. 56:4 (1903), 615644. MR 1511190 JFM 34.0461.02

[Epstein 1906] P. Epstein, "Zur Theorie allgemeiner Zetafunktionen, II", Math. Ann. 63:2 (1906), 205-216. MR 1511399 JFM 37.0433.02

[Gilkey 1995] P. B. Gilkey, Invariance theory, the heat equation, and the Atiyah-Singer index theorem, Studies in Advanced Mathematics, CRC Press, Boca Raton, FL, 1995. MR 98b:58156 Zbl 0856.58001

[Lang 1987] S. Lang, Elliptic functions, 2nd ed., Graduate Texts in Mathematics 112, Springer, New York, 1987. MR 88c:11028 Zbl 0615.14018

[Matsumoto 1998] K. Matsumoto, "Asymptotic series for double zeta, double gamma, and Hecke L-functions", Math. Proc. Cambridge Phil. Soc. 123:3 (1998), 385-405. MR 99i:11079 Zbl 0903. 11021 
[Ortenzi and Spreafico 2004] G. Ortenzi and M. Spreafico, "Zeta function regularization for a scalar field in a compact domain", J. Phys. A 37 (2004), 11499-11517. MR 2005k:81180 Zbl 1063.81083

[Quine and Choi 1996] J. R. Quine and J. Choi, "Zeta regularized products and functional determinants on spheres”, Rocky Mountain J. Math. 26:2 (1996), 719-729. MR 97k:58176 Zbl 0864.47024

[Rosenberg 1997] S. Rosenberg, The Laplacian on a Riemannian manifold, London Mathematical Society Student Texts 31, Cambridge University Press, Cambridge, 1997. MR 98k:58206 Zbl 0868.58074

[Shintani 1976] T. Shintani, "On evaluation of zeta functions of totally real algebraic number fields at non-positive integers", J. Fac. Sci. Univ. Tokyo Sect. IA Math. 23:2 (1976), 393-417. MR 55 \#266 Zbl 0349.12007

[Spreafico 2003] M. Spreafico, "Zeta functions and regularized determinants on projective spaces", Rocky Mountain J. Math. 33:4 (2003), 1499-1512. MR 2052503 (2004m:11138) Zbl 02104684

[Spreafico 2004] M. Spreafico, "On the non homogeneous quadratic Bessel zeta function", Mathematika 51 (2004). To appear.

[Spreafico 2005] M. Spreafico, "Zeta function and regularized determinant on a disc and on a cone", J. Geom. Phys. 54:3 (2005), 355-371. MR 2005k:11184 Zbl 1072.58025

[Spreafico 2006] M. Spreafico, "Zeta invariants for sequences of spectral type, special functions and the Lerch formula", Proc. Royal Soc. Edinb. (2006). To appear.

[Weil 1976] A. Weil, Elliptic functions according to Eisenstein and Kronecker, Springer, Berlin, 1976. MR 58 \#27769a Zbl 0318.33004

[Zagier 1975] D. Zagier, "A Kronecker limit formula for real quadratic fields", Math. Ann. 213 (1975), 153-184. MR 51 \#3123 Zbl 0283.12004

[Zagier 1977] D. Zagier, "Valeurs des fonctions zêta des corps quadratiques réels aux entiers négatifs", pp. 135-151 in Journées Arithmétiques de Caen (Caen (France), 1976), Astérisque 41-42, Soc. Math. France, Paris, 1977. MR 56 \#316 Zbl 0359.12012

Received July 9, 2002. Revised January 27, 2005.

\author{
MAURo SPREAFICO \\ Instituto de CiênCias Matemáticas e de ComputaÇão \\ UNIVERSIDADE DE SÃo PAULO \\ CAIXA POSTAL 668 \\ 13560-970 SÃo CARLOS, SP \\ BRAZIL \\ mauros@icmc.usp.br
}

